\title{
Controle de Ipomoea hederifolia com baixo volume de calda com e sem adjuvante aplicada com bico de energia centrífuga ${ }^{1}$
}

\section{Ipomoea hederifolia control with low volume using adjuvant spray liquid with spray rotary nozzle}

\author{
Micheli Satomi Yamauti ${ }^{2 *}$, Paulo Roberto Fidelis Giancotti ${ }^{2}$, Bruno Nascimento Lodo ${ }^{3}$, Lonjoré \\ Leocadio de Lima ${ }^{4}$, Marcelo da Costa Ferreira ${ }^{5}$
}

\begin{abstract}
Resumo - O emprego de conhecimentos científicos que possibilitem a caracterização e a escolha do bico de pulverização adequado para o controle do alvo biológico é função da tecnologia de aplicação. Objetivou-se caracterizar a distribuição volumétrica, o espaçamento entre bicos, o diâmetro e a uniformidade de gotas produzidas por um bico de pulverização de energia centrífuga e o controle em pós-emergência de Ipomoea hederifolia utilizando-se o herbicida diuron + hexazinone $\left(1170+330\right.$ g i.a. ha $\left.{ }^{-1}\right)$, com volumes de aplicação de 20 e $40 \mathrm{~L}$ $\mathrm{ha}^{-1}$, com a adição ou não de adjuvantes (organo siliconado - 0,025\% v/v (Silwett ${ }^{\circledR}$ ) e óleo vegetal - $1 \% \mathrm{v} / \mathrm{v}\left(\right.$ Veget $\left.\mathrm{Oil}^{\circledR}\right)$ ). Os tratamentos com uso de bico rotativo modelo Herbi-4 ${ }^{\circledR}$ foram comparados a testemunha com ponta hidráulica TT110.01 (150 L ha $\left.{ }^{-1}\right)$. Foram avaliados o controle das plantas daninhas, o diâmetro médio volumétrico (DMV), a porcentagem de gotas com diâmetro inferior a $100 \mu \mathrm{m}(\% 100 \mu \mathrm{m})$ e a amplitude relativa (AR). A adição de adjuvantes à calda de pulverização não promoveu acréscimo no controle de $I$. hederifolia. $\mathrm{O}$ bico rotativo de energia centrífuga reduziu consideravelmente o risco de deriva em relação à ponta TT110.01, devido ao aumento do DMV, redução da $\% 100 \mu \mathrm{m}$ e maior uniformidade das gotas produzidas, apesar de apresentar padrão de distribuição desuniforme. Não houve diferença de controle de $I$. hederifolia entre o bico bico rotativo Herbi- ${ }^{\circledR}$ e ponta hidráulica TT110.01.
\end{abstract}

Palavras-chave: bico rotativo, espectro de gotas, volume de aplicação, pulverização.

Abstract - The use of scientific knowledge that make possible the characterization and choice of spray nozzle suitable to control of biological target is a function of application technology. This work aimed to characterize the volumetric distribution, the spacing between nozzles, the diameter and uniformity of droplets produced by spraying nozzle of centrifugal energy and control in post-emergence of $I$. hederifolia with herbicide diuron + hexazinone $\left(1170 \mathrm{~g} \mathrm{ha}^{-1}+330\right.$ $\mathrm{g} \mathrm{ha}^{-1}$ ) using spray volumes of 20 and $40 \mathrm{~L} . h a^{-1}$ with or without addition of adjuvants

\footnotetext{
* Autor para correspondência

${ }^{1}$ Recebido para publicação em 10/01/2012 e aceito20/04/2012.

2 Eng. Agr. MSc Doutoranda(o), Depto. de Biologia Aplicada a Agropecuária, FCAV/Unesp, Jaboticabal-SP, micheliyamauti@yahoo.com.br; paulogiancotti@gmail.com

${ }^{3}$ Eng. Agr. MSc., Depto. de Fitossanidade, FCAV/Unesp, Jaboticabal-SP, brunolodo@ yahoo.com.br;

${ }_{5}^{4}$ Eng. Agr. MSc. Doutoranda, Depto. de Fitossanidade, FCAV/Unesp, Jaboticabal-SP, lonjore@ hotmail.com;

5 Prof. Adjunto do Depto. De Fitossanidade, FCAV/Unesp, Jaboticabal-SP, mdacosta@ fcav.unesp.br;
} 
(organosilicone - 0,025\% v/v (Silwett) and vegetable oil - 1\% v/v (Veget Oil). Treatments with use of rotative jet Herbi- $4^{\circledR}$ model were compared to the check with hydraulic nozzle TT110.01 $\left(150 \mathrm{~L} \mathrm{ha}^{-1}\right)$. Weeds control, volumetric median diameter (VMD), the percentage of droplets below $100 \mu \mathrm{m}(\% 100 \mu \mathrm{m})$ diameter and relative amplitude (RA) were evaluated. The addition of adjuvants to spray solution did not increase $I$. hederifolia control. The nozzle of centrifugal energy (model Herbi-4) has significantly reduced the risk of drift towards the use of hydraulic nozzle TT110.01, due to increase diameter of droplets, reducing the percentage of droplets less than $100 \mu \mathrm{m}$ and greater uniformity of droplet size, despite a pattern of distribution to be no uniform. There was no difference in I. hederifolia control between rotative jet Herbi- $4^{\circledR}$ and hidraulic nozzles TT110.01.

Key-Words: atomizer, droplet spectrum, application volume, spray distribution.

\section{Introdução}

As pontas de pulverização são consideradas os principais componentes da pulverização, pois possuem características que asseguram melhor segurança e efetividade no controle de pragas, doenças e plantas daninhas (Viana et al., 2010). A correta aplicação do produto fitossanitário é possível quando se dispõe de pontas que propiciem distribuição transversal uniforme, espectro de gotas uniforme e de tamanho adequado (Cunha \& Silva, 2010).

No caso de bicos de energia centrífuga ou bicos rotativos existem basicamente duas formas de aplicação: a primeira é a utilização de alta rotação para produzir gotas pequenas que são lançadas sobre a área a ser tratada e a segunda é a formação de gotas maiores, para aplicação sem deriva. O padrão de deposição dos bicos rotativos é bastante típico, sendo o seu conhecimento, imprescindível para efetuar a correta sobreposição das faixas, estabelecendo a correta distância entre bicos ou espaçamento entre as diversas passadas de um bico (Matuo, 1990).

Os fatores que influenciam o espectro de gotas produzidas por diferentes bicos de pulverização são tipo de bico quanto à energia de formação das gotas, vazão nominal, pressão do líquido, propriedades da calda (Womac et al., 1999), entre outros. Por isso é preciso conhecer as características técnicas dos tipos de bico, visando a sua correta seleção e, com isso, aplicações mais eficientes e seguras (Cunha et al., 2004).

O uso de adjuvantes possibilita minimizar os efeitos do ambiente que podem comprometer a eficiência de um tratamento fitossanitário (Carbonari et al., 2005). Contudo, grande parte dos problemas do uso dos mesmos origina-se do desconhecimento de sua ação e das implicações de seu emprego na eficiência de controle do alvo (Antuniassi, 2006), inclusive em relação ao espectro de gotas gerado. Segundo Cunha et al. (2010) pouca informação científica existe sobre o assunto, dificultando a seleção ou a recomendação dos adjuvantes.

A uniformidade de distribuição volumétrica da calda serve de referência para verificar a distribuição do ingrediente ativo no alvo, e essa uniformidade é alterada pelo espaçamento entre pontas, pressão de trabalho, altura da barra em relação ao alvo e ângulo de abertura do jato das pontas de pulverização. A uniformidade da distribuição é aferida pelo coeficiente de variação da sobreposição da deposição de um conjunto de bicos em uma barra (Bauer \& Raetano, 2004). A uniformidade de distribuição preconizada pela norma UNE-EN 12761:2 (2002), para modelos 
de energia hidráulica, estabelece que $\mathrm{o}$ as leituras de dois milisegundos, sendo coeficiente de variação $(\mathrm{CV} \%)$ da realizadas 500 leituras por segundo. $\mathrm{O}$ bico de sobreposição de jatos seja menor que $7 \%$ pulverização foi posicionado a $0,4 \mathrm{~m}$ do feixe quando utilizados altura, espaçamento e do laser para que as gotas produzidas pressão recomendados pelo fabricante e de até atravessassem o feixe.

$9 \%$ em configuração distinta.

A determinação do espectro de gotas produzidas pelas pontas de pulverização é imprescindível para a aplicação de herbicidas, pois a partir dessa informação, efetua-se a escolha da ponta de acordo com o potencial de deriva, características do herbicida e os riscos de volatilização e escorrimento de calda nas folhas das plantas daninhas (Viana et al., 2010). Diversos trabalhos têm mostrado que aplicações de herbicidas em pós-emergência nos estádios iniciais de crescimento das plantas daninhas resultam em controle mais eficiente do que aplicações efetuadas mais tardiamente, devido ao maior desenvolvimento vegetativo e tolerância aos herbicidas nesses casos (Fleck et al., 2008).

O objetivo deste trabalho foi avaliar o controle de Ipomoea hederifolia com baixo volume de calda aplicada com e sem adjuvantes, utilizando bico de energia centrífuga.

\section{Material e Métodos}

Avaliação do espectro e caracterização de gotas.

A análise do espectro de gotas foi realizada de forma direta, utilizando-se de analisador de partículas (Mastersizer $\mathrm{S}^{\circledR}$, Malvern Instruments Co.), com a lente focal de $300 \mathrm{~mm}$, para partículas de 0,5 a $900 \mathrm{~mm}$, que se baseia na difração da luz após o choque com as gotas, em que o diâmetro das gotas é proporcional ao ângulo do desvio sofrido pelo raio laser (Etheridge et al., 1999). No analisador, foram realizadas três análises de 1,5 s para cada tratamento, com intervalo entre
Avaliou-se o diâmetro das gotas, tal que $10 \%$ do volume aplicado seja inferior a esse valor (Dv 0,1); o diâmetro de gotas, tal que $50 \%$ do volume aplicado seja inferior a esse valor (Dv 0,5); o diâmetro de gotas, tal que $90 \%$ do volume aplicado seja inferior a esse valor (Dv 0,9); o coeficiente de uniformidade (AR) e a porcentagem do volume aplicado cujas gotas possuam diâmetro inferior a 100 $\mu \mathrm{m}(\% \mathrm{~V}<100 \mu \mathrm{m})$.

O coeficiente de uniformidade foi dado pela equação: $A R=\frac{D \nu 0,9-D v 0,1}{D \nu 0,5}$

O delineamento utilizado foi o inteiramente casualizado, em esquema fatorial $2 \times 3$ com três repetições. O primeiro fator representou o volume de aplicação $\left(20 \mathrm{~L} \mathrm{ha}^{-1} \mathrm{e}\right.$ $40 \mathrm{~L} \mathrm{ha}^{-1}$ ), o segundo os adjuvantes (organo silicone $-0,025 \% \mathrm{v} / \mathrm{v}\left(\right.$ Silwett $\left.{ }^{\circledR}\right)$, óleo vegetal - $1 \%$ v/v (Veget $\mathrm{Oil}^{\circledR}$ ) e ausência de adjuvante) e também foi analisada a testemunha, representada pela ponta hidráulica TT110.01 (150 L ha ${ }^{-1}$ e pressão de $\left.200 \mathrm{kPa}\right)$.

Os dados de Dv 0,1, Dv 0,5, Dv 0,9, porcentagem de gotas com diâmetro igual ou inferior a $100 \mu \mathrm{m}$ e a amplitude relativa foram submetidos à análise de variância e as médias comparadas pelo teste de Tukey $(\mathrm{P}<0,05)$.

\section{Caracterização do perfil de distribuição volumétrica dos jatos pulverizados}

Para a determinação do perfil de distribuição volumétrica das caldas pulverizadas, utilizou-se o bico hidráulico com ponta TT-110.01 como testemunha a pressão de $200 \mathrm{kPa}$. Utilizou-se mesa de provas construída de acordo com as especificações da 
Organización Mundial de la Salud (1976) para testes de bicos, constituída de 67 canaletas de metal corrugado com $2,5 \mathrm{~cm}$ de largura cada, totalizando $167,5 \mathrm{~cm}$ de largura por $100 \mathrm{~cm}$ de comprimento. As canaletas possuem inclinação de $15^{\circ}$ para o líquido pulverizado escoar até tubos de ensaio graduados, utilizados como coletores. A ponta hidráulica TT110.01 (150 L ha ${ }^{-1}$ e pressão de $200 \mathrm{kPa}$ ) foi posicionada verticalmente à altura da mesa de $50 \mathrm{~cm}$, acima da canaleta $\mathrm{n}^{\mathrm{o}} 33$, no centro da mesa de provas.

Para a avaliação do bico rotativo a mesa de provas não pôde ser utilizada, pois parte do jato extrapolava a região de coleta, comprometendo a capacidade de leituras. Portanto, para a avaliação da distribuição deste tipo de bico, foram utilizados papéis hidrossensíveis como coletores. Os papéis foram dispostos um ao lado do outro, espaçados de $20 \mathrm{~cm}$ entre si, totalizando uma faixa de 2,40 m, em três repetições. Após a passagem do aplicador, os amostradores foram retirados e colocados em sacos de papel identificados, com a finalidade de mantê-los íntegros e fora da exposição de umidade. Após a aplicação, os papéis hidrossensíveis foram digitalizados em escâner de mesa, com resolução de 300 dpi. As imagens obtidas foram analisadas no software QUANT v 0.1, para quantificação da porcentagem da área coberta pelas gotas (Vale et al., 2001).

Para a determinação do espaçamento de trabalho entre bicos, foram utilizados os coeficientes de variação, dentro do limite aceitável, assumido como parâmetro o valor de $10 \%$, que é aceito pela FAO (1997).

$\mathrm{O}$ delineamento utilizado foi $\mathrm{o}$ inteiramente casualizado, em esquema fatorial $2 \times 3+1$ com três repetições. $\mathrm{O}$ primeiro fator representou o volume de aplicação $\left(20 \mathrm{~L} \mathrm{ha}^{-1} \mathrm{e}\right.$ $40 \mathrm{~L} \mathrm{ha}^{-1}$ ), o segundo os adjuvantes (organo silicone $-0,025 \% \mathrm{v} / \mathrm{v}\left(\right.$ Silvett $\left.^{\circledR}\right)$, óleo vegetal $1 \%$ v/v (Veget $\mathrm{Oil}^{\circledR}$ ) e ausência de adjuvante) e também foi analisada a testemunha, representada pela ponta hidráulica TT110.01 (150 L ha ${ }^{-1}$ e pressão de $200 \mathrm{kPa}$ ).

Os dados obtidos foram submetidos à análise de variância e as médias comparadas pelo teste de Tukey $(\mathrm{P}<0,05)$. Realizou-se análise de regressão com os dados da variação do volume aplicado, com a finalidade de verificar qual equação de melhor ajuste aos dados para determinação do espaçamento entre bicos.

\section{Eficácia de controle da planta daninha.}

O ensaio foi conduzido no ano agrícola de 2009/2010. Para isso foram mantidas cinco plantas de I. hederifolia em vasos com capacidade de $3 \mathrm{~L}$, preenchidos com substrato composto por solo, areia e esterco na proporção de $1: 1: 1$.

O delineamento utilizado foi o inteiramente casualizado, em esquema fatorial $2 \times 3+2$ testemunhas, com 4 repetições. Foi realizada aplicação dos herbicidas diuron + hexazinone $\left(\right.$ Dizone $\left.^{\circledR}\right)\left(1170+330\right.$ g i.a. ha $\left.^{-1}\right)$, em dois volumes de aplicação $\left(20 \mathrm{~L} \mathrm{ha}^{-1}\right.$ e 40 $\mathrm{L} \mathrm{ha}^{-1}$ ), com adição de adjuvantes a calda de pulverização: organosilicone $\left(\right.$ Silwett $\left.^{\circledR}\right)$ a $0,025 \% \mathrm{v} / \mathrm{v}$ ou óleo vegetal $\left(\mathrm{Veget} \mathrm{Oil}^{\circledR}\right.$ ) a $1,0 \% \mathrm{v} / \mathrm{v}$ e uma testemunha sem adição de adjuvante. Utilizou-se um bico de pulverização rotativo modelo Herbi - $4^{\circledR}$ (energia centrífuga), que possui rotação de 2000 RPM e vazão de $100 \mathrm{~mL} \mathrm{~min}^{-1}$, sendo a pressão exercida por gravidade, e como testemunha representada pela ponta de energia hidráulica TT110.01 (150 L ha ${ }^{-1}$ ) e outra sem aplicação.

A aplicação foi realizada em pósemergência inicial (2 a 3 folhas verdadeiras), na ocasião as condições climáticas eram de temperatura de $27{ }^{\circ} \mathrm{C}$, umidade relativa do ar de $62 \%$ e velocidade do vento de $3,5 \mathrm{~km} \mathrm{~h}^{-1}$. 
Foram avaliadas as porcentagens de controle por meio de uma escala de observação visual de notas de $0 \%$ a $100 \%$, onde $0 \%$ corresponde a nenhum controle e $100 \%$ ao controle total da planta daninha, aos 7, 14 e 21 dias após a aplicação (DAA). Também foram avaliadas a massa seca da parte aérea e da raiz aos 21 DAA, ocasião em que as plantas foram separadas em raízes e parte aérea, sendo posteriormente acondicionadas em sacos de papel e levadas à estufa de circulação forçada de ar à $65 \pm 5^{\circ} \mathrm{C}$ por um período de 72 horas.

Os dados de porcentagem de controle e massa seca da parte aérea e da raiz foram submetidos à análise de variância e as médias comparadas pelo teste Tukey $(\mathrm{P}<0,05)$.

\section{Resultados e Discussão}

\section{Avaliação do espectro e caracterização de gotas}

Observa-se que não houve diferença significativa entre os volumes de aplicação para as variáveis DV 0,1 e $\% \mathrm{~V}<100 \mu \mathrm{m}$. Entretanto, verificou-se diferença significativa entre os volumes de aplicação e para Dv 0,5, Dv 0,9 e AR, sendo que o volume de aplicação de $40 \mathrm{~L} \mathrm{ha}^{-1}$ promoveu aumento no diâmetro das gotas e maior valor de AR, o que significa uma menor uniformidade (Tabela 1).

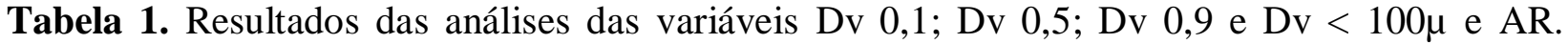
Jaboticabal, 2009.

\begin{tabular}{cccccc}
\hline \multirow{2}{*}{ Fatores } & Dv 0,1 & Dv 0,5 & Dv 0,9 & $\% \mathrm{~V}<100 \mu \mathrm{m} \%$ & $\mathrm{AR}$ \\
\cline { 2 - 6 } & \multicolumn{7}{c}{ Volume (V) } \\
\hline 20 L & $149,5 \mathrm{~A}$ & $229,2 \mathrm{~B}$ & $350,8 \mathrm{~B}$ & $1,0 \mathrm{~A}$ & $0,8 \mathrm{~B}$ \\
$40 \mathrm{~L}$ & $152,5 \mathrm{~A}$ & $259,6 \mathrm{~A}$ & $417,5 \mathrm{~A}$ & $1,2 \mathrm{~A}$ & $1,0 \mathrm{~A}$ \\
\hline \multicolumn{7}{c}{ Adjuvantes $(\mathrm{A})$} \\
\hline Ausente & $139,8 \mathrm{~B}$ & $213,7 \mathrm{~B}$ & $329,8 \mathrm{~B}$ & $1,5 \mathrm{~A}$ & $0,9 \mathrm{~A}$ \\
Org. Silic. & $154,6 \mathrm{~A}$ & $256,2 \mathrm{~A}$ & $409,0 \mathrm{~A}$ & $1,0 \mathrm{AB}$ & $0,9 \mathrm{~A}$ \\
Oleo & $158,8 \mathrm{~A}$ & $263,4 \mathrm{~A}$ & $413,6 \mathrm{~A}$ & $0,9 \mathrm{~B}$ & $1,0 \mathrm{~A}$ \\
\hline $\mathrm{F}_{\mathrm{v}}$ & $0,9^{\mathrm{NS}}$ & $11,6^{* *}$ & $10,8^{* *}$ & $1,5^{\mathrm{NS}}$ & $15,1^{* *}$ \\
$\mathrm{~F}_{\mathrm{A}}$ & $13,0^{* *}$ & $12,5^{* *}$ & $7,2^{*}$ & $3,2^{*}$ & $1,1^{\mathrm{NS}}$ \\
VxA & $10,6^{* *}$ & $21,1^{* *}$ & $22,4^{* *}$ & $1,3^{\mathrm{NS}}$ & $18,2^{* *}$ \\
CV $(\%)$ & 4,5 & 7,7 & 11,2 & 12,6 & 9,5 \\
\hline
\end{tabular}

Médias seguidas de mesma letra não diferem entre si, pelo teste Tukey, a $5 \%$ de probabilidade; **: significativo $(\mathrm{P}<0,01)$; *: significativo $(\mathrm{P}<0,05)$; NS: não-significativo; $\mathrm{C}$.V.: coeficiente de variação.

Verifica-se que independente do adjuvante adicionado à calda de pulverização, houve diferença significativa em relação a não adição de adjuvante para todas as características avaliadas, com exceção da \%V $<100 \mu \mathrm{m}$, em que a adição do organosilicone não resultou em diferença significativa. Entre os adjuvantes avaliados não se verificou diferença significativa para as características estudadas.

Realizou-se a avaliação das mesmas características em um bico hidráulico (TT110.01) utilizado como testemunha, cujos resultados médios encontrados foram $\mathrm{Dv} 0,1=$ 73,99 , Dv 0,5 = 168,33, Dv 0,9 = 339,19, \%V $<100 \mu \mathrm{m}=20,67 \%$ e AR. $=1,57$. Tais resultados foram semelhantes aos encontrados 
por Camara et. al. (2008) avaliando o espectro de gotas de bicos hidráulicos.

Segundo Cunha et al. (2004), gotas com diâmetro inferior a $100 \mu \mathrm{m}$ são as principais responsáveis pelo aumento no risco da deriva. Quanto menor essa porcentagem, menor o risco de deriva durante a aplicação. Não existe valor padrão indicativo de risco de deriva ou de aplicação segura (Cunha et al., 2010). Em geral, valores inferiores a $15 \%$ do volume pulverizado composto por gotas com diâmetro inferior a $100 \mu \mathrm{m}$ parecem ser mais adequados a uma aplicação segura (Cunha et al., 2003). A utilização do bico rotativo Herbi - $4^{\circledR}$ manteve os valores de $\% \mathrm{~V}<100 \mu \mathrm{m}$ entre 0,9 e $1,5 \%$, diminuindo consideravelmente o risco de deriva. Já os valores encontrados para ponta de energia hidráulica TT110.01 (150 $\mathrm{L} \mathrm{ha}^{-1}$ ) foram acima de $20 \%$, estando mais sujeito a deriva, e com isso sua utilização deve ser criteriosa, evitando-se aplicações com velocidade do vento elevada.

A utilização dos adjuvantes à calda de pulverização aumentou o diâmetro das gotas, representado por Dv 0,1, Dv 0,5 e Dv 0,9, indicando que sua utilização pode reduzir o risco de deriva na pulverização, uma vez que quanto maior o diâmetro das gotas menor é o risco de deriva. Cunha \& Alves (2009), estudando as propriedades físico-químicas de caldas de pulverização com alguns adjuvantes, comentaram que, em geral, a elevação da viscosidade da calda com adjuvantes está associada à geração de gotas de pulverização maiores, explicando assim o ocorrido no experimento.

O uso do bico rotativo Herbi $-4^{\circledR}$ diminuiu o AR em relação à utilização da ponta hidráulica TT110.01, indicando menor variação no espectro de tamanho de gotas, e consequentemente, maior uniformidade (Tabela 1). Di Oliveira et al. (2010), também encontraram que o bico rotativo produz gotas de maior uniformidade com menor porcentagem de gotas suscetíveis a deriva.

Verifica-se que para as variáveis Dv 0,1, Dv 0,5 e Dv 0,9 a associação do adjuvante organo silicone com o volume de aplicação de $40 \mathrm{~L} \mathrm{ha}^{-1}$ aumentou o diâmetro das gotas. A associação de óleo vegetal Veget $\mathrm{Oil}^{\circledR}(1,0 \%$ v/v) com o volume de aplicação de $20 \mathrm{~L} \mathrm{ha}^{-1}$ promoveu a menor $\mathrm{AR}$, reduzindo a variação no espectro de gotas, e aumentando a uniformidade (Tabela 2).

A distribuição volumétrica foi afetada pela adição de adjuvantes tanto para o volume de aplicação de $20 \mathrm{~L} \mathrm{ha}^{-1}$ quanto para $40 \mathrm{~L} \mathrm{ha}^{-1}$, e diferiu da testemunha convencional (Figura 1). Para o volume de aplicação de $20 \mathrm{~L} \mathrm{ha}^{-1}$ houve uma maior deposição com a adição do organo siliconado e uma diminuição com a adição de óleo vegetal, ocorrendo aumento na distribuição nas posições mais distantes do centro (extremidades). Para o volume de $40 \mathrm{~L}$ $\mathrm{ha}^{-1}$ a distribuição foi semelhante com a adição do organo siliconado, ocorrendo uma pequena redução com o uso de óleo vegetal e menor deposição nas extremidades. As distribuições volumétricas com uso do bico rotativo apresentaram diferenças se comparadas a testemunha convencional, onde se utilizou a ponta hidráulica TT110.01. Segundo Cunha et al. (2010), o efeito dos adjuvantes é dependente da ponta de pulverização e do produto empregado. 
Tabela 2. Interação entre os fatores (Coef.de aplicação) para as variáveis Dv 0,1; Dv 0,5; Dv 0,9 e AR. Jaboticabal, 2009.

\begin{tabular}{|c|c|c|c|c|}
\hline \multirow[b]{3}{*}{ Volume (L) } & \multicolumn{3}{|c|}{ Adjuvantes } & \multirow[t]{3}{*}{ Médias } \\
\hline & Ausente & Org. Silic. & Óleo Vegetal & \\
\hline & \multicolumn{3}{|c|}{ Dv $0,1(\mu \mathrm{m})$} & \\
\hline 20 & $136,92 \mathrm{bA}$ & $144,82 \mathrm{bB}$ & $166,89 \mathrm{aA}$ & 149,54 \\
\hline 40 & $142,61 \mathrm{bA}$ & $164,29 \mathrm{aA}$ & $150,62 \mathrm{abA}$ & 152,51 \\
\hline \multirow[t]{2}{*}{ Médias } & 139,76 & 154,55 & 158,76 & \\
\hline & \multicolumn{3}{|c|}{ Dv $0,5(\mu \mathrm{m})$} & \\
\hline 20 & $188,96 \mathrm{bA}$ & $211,27 \mathrm{bB}$ & $287,52 \mathrm{aA}$ & 229,25 \\
\hline 40 & $238,35 \mathrm{bA}$ & $301,13 \mathrm{aA}$ & $239,30 \mathrm{bA}$ & 259,60 \\
\hline \multirow[t]{2}{*}{ Médias } & 213,66 & 256,2 & 263,41 & \\
\hline & \multicolumn{3}{|c|}{ Dv $0,9(\mu \mathrm{m})$} & \\
\hline 20 & $279,75 \mathrm{bA}$ & $302,08 \mathrm{bB}$ & $470,33 \mathrm{aA}$ & 350,82 \\
\hline 40 & $379,93 \mathrm{bA}$ & $515,67 \mathrm{aA}$ & $365,96 \mathrm{bA}$ & 417,52 \\
\hline \multirow[t]{2}{*}{ Médias } & 329,84 & 409,03 & 413,65 & \\
\hline & \multicolumn{3}{|c|}{$\mathrm{AR}$} & \\
\hline 20 & $0,76 \mathrm{bA}$ & $0,75 \mathrm{bB}$ & $1,04 \mathrm{aA}$ & 0,85 \\
\hline 40 & $0,99 \mathrm{aA}$ & $1,16 \mathrm{aA}$ & $0,86 \mathrm{aA}$ & 1,01 \\
\hline Médias & 0,88 & 0,95 & 0,96 & \\
\hline
\end{tabular}

Médias seguidas de mesma letra minúscula nas linhas e maiúscula nas colunas não diferem entre si, pelo Teste Tukey, a 5\% de probabilidade.
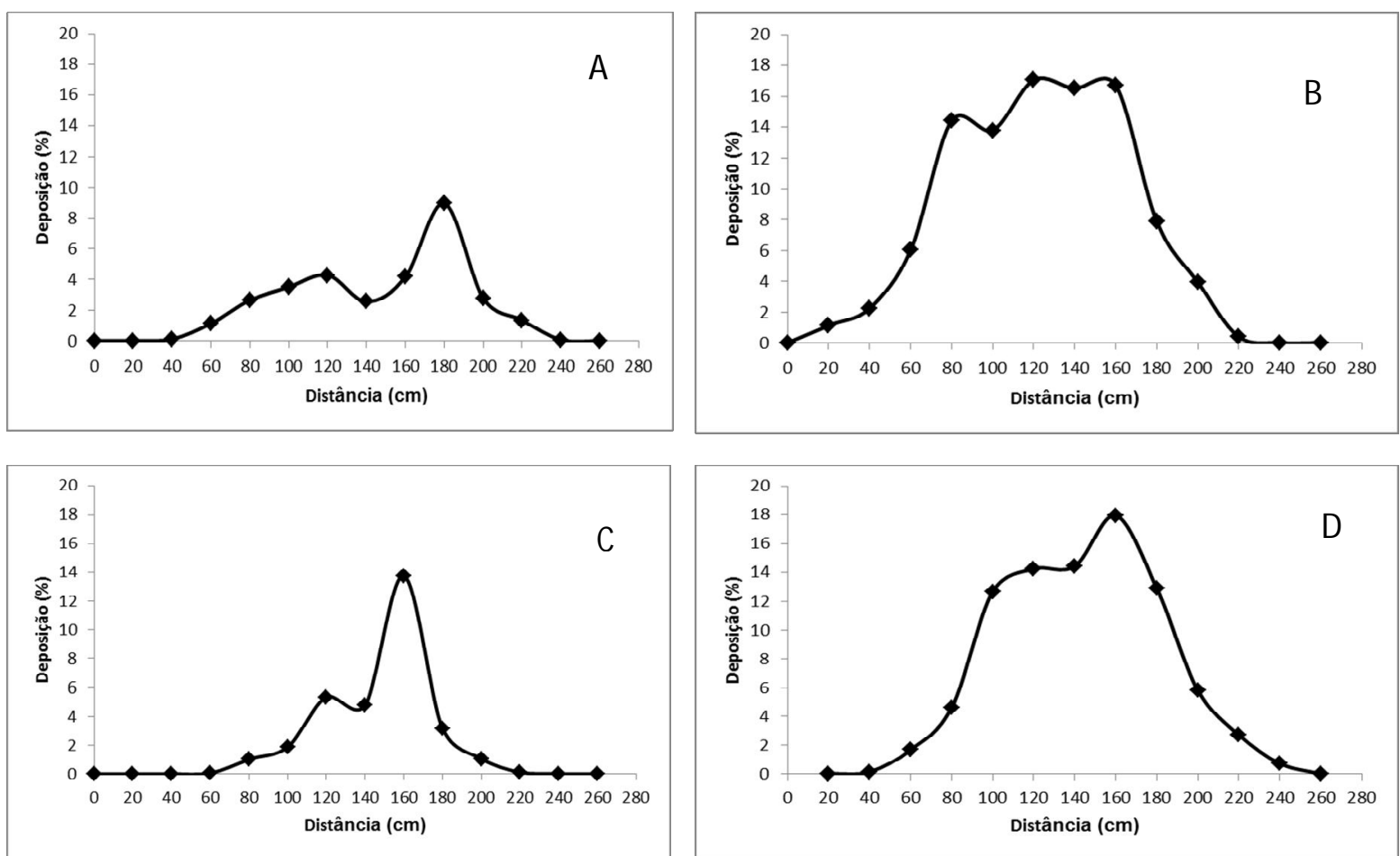

Rev. Bras. Herb., v.11, n.1, p.49-61, jan./abr. 2012 

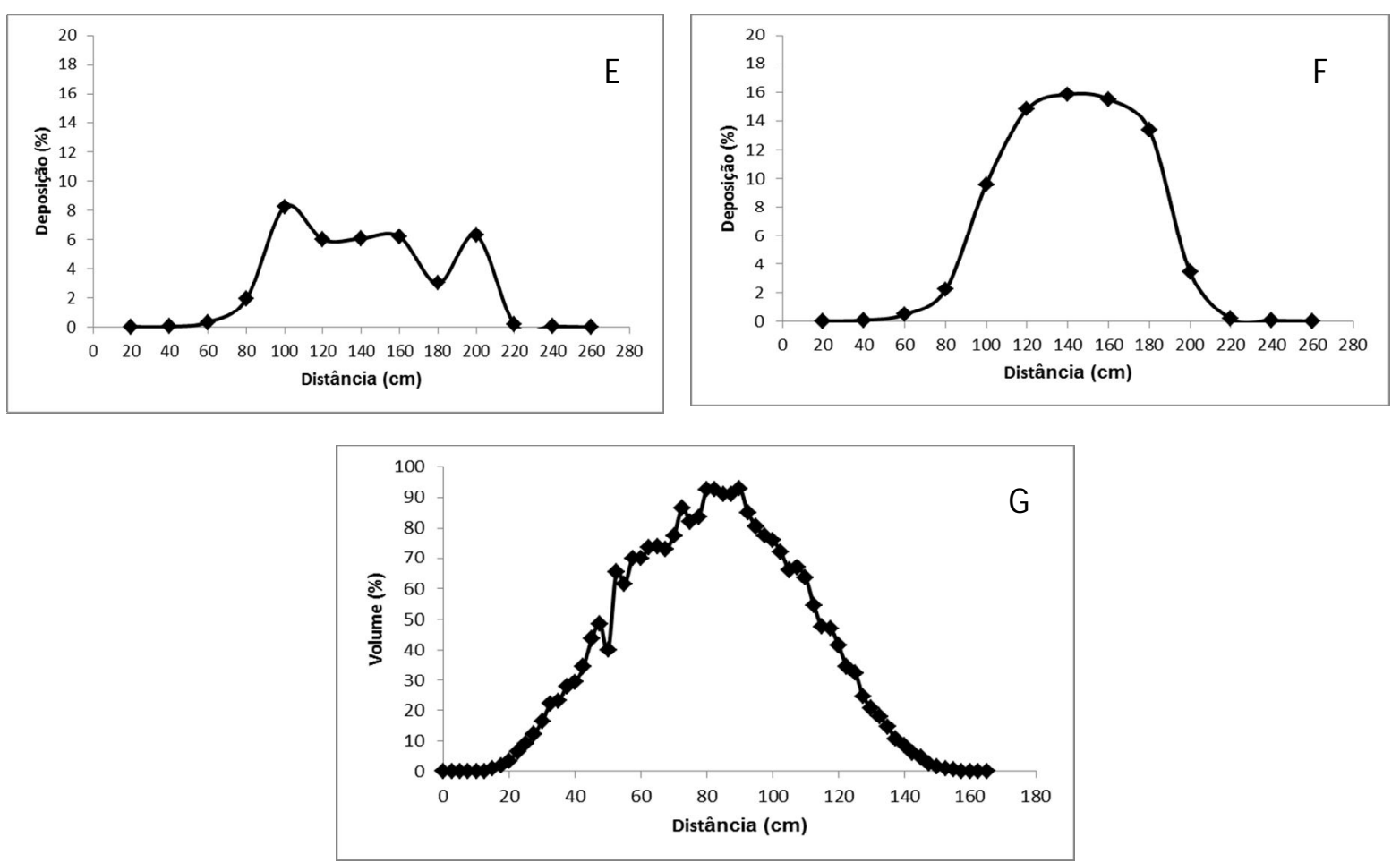

Figura 1. Perfil de distribuição volumétrica sem adjuvante com volume de aplicação de $20 \mathrm{~L} \mathrm{ha}^{-1}$ (A) e 40 $\mathrm{L} \mathrm{ha}^{-1}(\mathrm{~B})$, com adição de organo siliconado com $20 \mathrm{~L} \mathrm{ha}^{-1}$ (C) e $40 \mathrm{~L} \mathrm{ha}^{-1}$ (D), com óleo vegetal $20 \mathrm{~L} \mathrm{ha}^{-1}$ (E) e $40 \mathrm{~L} \mathrm{ha}^{-1}(\mathrm{~F})$ e testemunha convencional utilizando ponta hidráulica TT110.01 (G). Jaboticabal, 2009.

\section{Caracterização do perfil de distribuição volumétrica dos jatos pulverizados}

A distribuição das gotas produzidas pelo bico rotativo Herbi $-4^{\circledR}$, de maneira geral, resultou em padrão de distribuição desuniforme. Tal fato pode ser explicado devido às oscilações no momento da aplicação, uma vez que esta operação foi realizada manualmente. Verifica-se que o mesmo não ocorreu para testemunha ponta hidráulica TT110.01, uma vez que por ter sido fixada a mesa de distribuição, esteve menos sujeita a oscilações que resultou em padrão de distribuição uniforme (Figura 1).

Quando utilizando o volume de aplicação de $20 \mathrm{~L} \mathrm{ha}^{-1} \mathrm{o}$ espaçamento entre bicos foi de 40,6 cm com o uso de óleo vegetal. Já na ausência de adjuvante e com o uso de organo silicone os valores dos espaçamentos foram de 29 e $22 \mathrm{~cm}$, respectivamente. Já com o volume de aplicação de $40 \mathrm{~L} \mathrm{ha}^{-1}$ os espaçamentos variaram chegando a $90 \mathrm{~cm}$ quando não se adicionou adjuvante a calda de pulverização (Figura 3). 

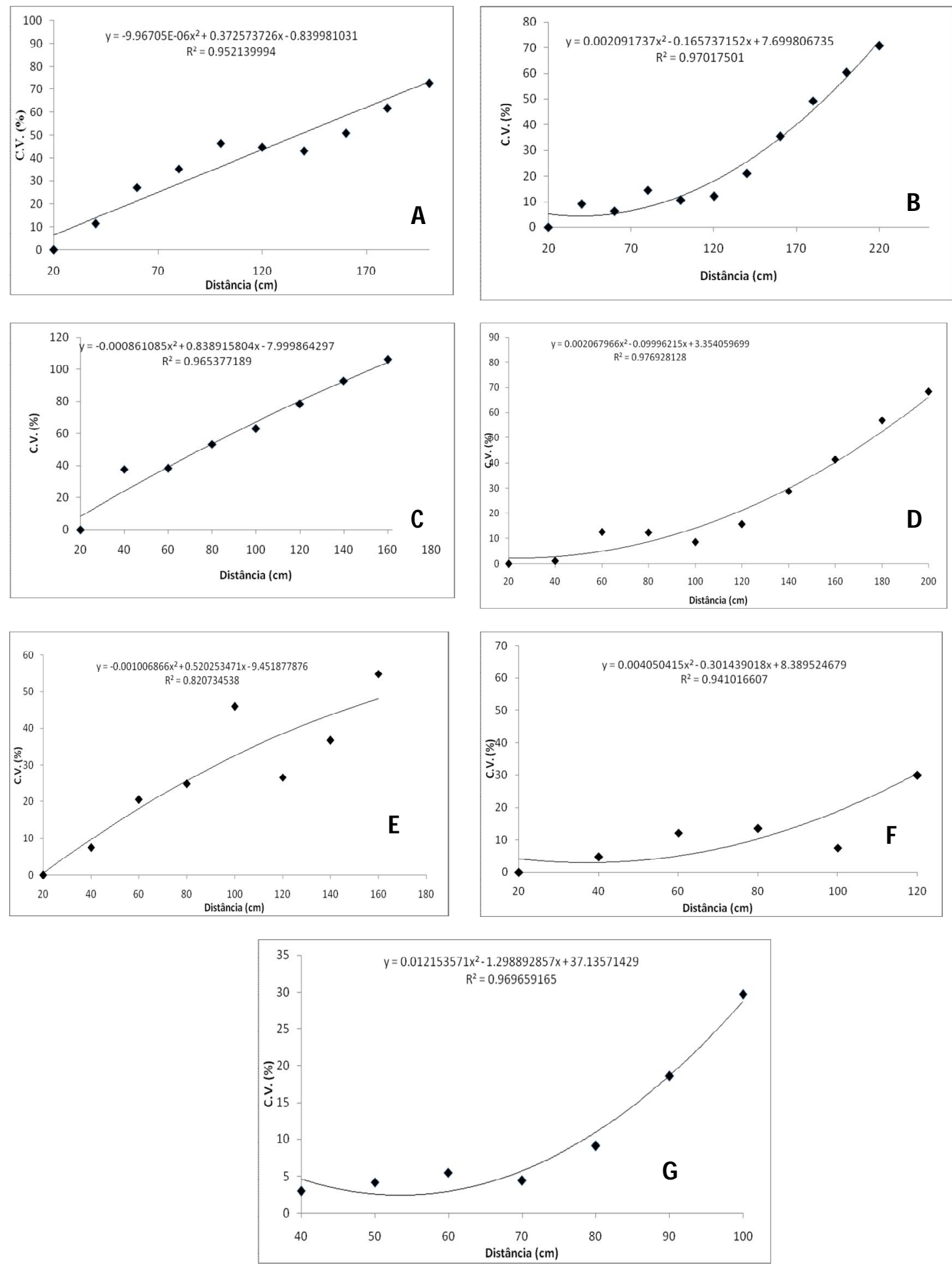

Figura 2. Equações de regressão para a determinação do espaçamento entre bicos, em função do coeficiente de variação para volume de aplicação de $20 \mathrm{~L} \mathrm{ha}^{-1}$ (A) e $40 \mathrm{~L} \mathrm{ha}^{-1}$ (B), com adição de organo silicone com $20 \mathrm{~L} \mathrm{ha}^{-1}$ (C) e $40 \mathrm{~L} \mathrm{ha}^{-1}$ (D), com óleo vegetal $20 \mathrm{~L} \mathrm{ha}^{-1}$ (E) e $40 \mathrm{~L}$ ha $^{-1}$ (F) e testemunha convencional utilizando ponta hidráulica TT110.01 (G). Jaboticabal, 2009. 


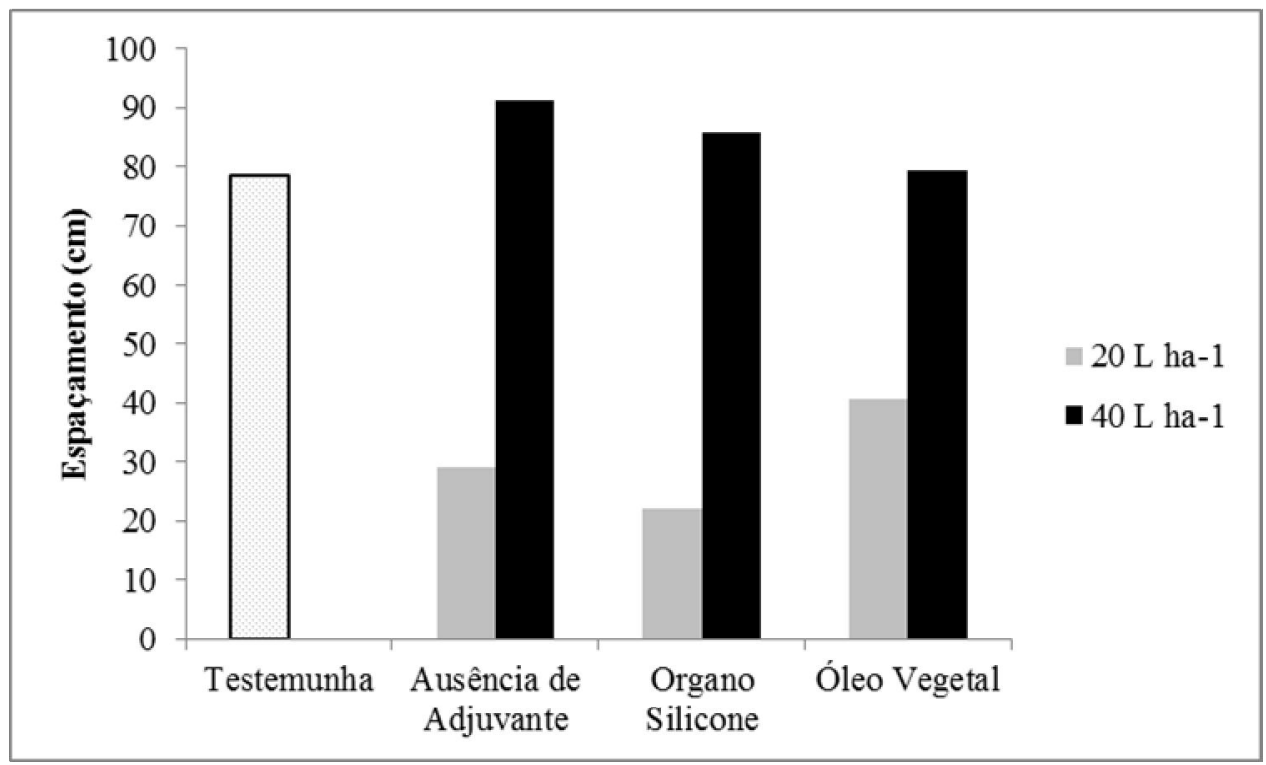

Figura 3. Espaçamento entre bicos aceitáveis (com C.V. de 10\%) utilizando-se diferentes volumes de aplicação e adjuvantes. Jaboticabal, 2009.

Como o experimento foi desenvolvido em condições de laboratório, os espaçamentos entre bicos foram calculados considerando os coeficientes de variação aceitáveis na literatura. Portanto, inferiores a $10 \%$, uma vez que, em condições de campo, tais valores tendem a aumentar, seja pelas condições meteorológicas inerentes por ocasião da aplicação, ou mesmo pelos movimentos desordenados da barra de pulverização (Perecin et al., 1998).

\section{Eficácia de controle da planta daninha.}

A porcentagem de controle de $I$. hederifolia aos 7 DAA foi maior quando utilizou-se óleo vegetal e o organo silicone com volume de aplicação de $40 \mathrm{~L} \mathrm{ha}^{-1}$. Porém, não diferiu significativamente da testemunha com ponta hidráulica (TT110.01).
Aos 7 DAA a porcentagem de controle foi maior quando o volume de aplicação foi de $40 \mathrm{~L} \mathrm{ha}^{-1}$ se comparado ao volume de $20 \mathrm{~L} \mathrm{ha}^{-}$ 1. Já aos 14 e 21 dias não se verificou diferença significativa entre os tratamentos, e o controle para todos os tratamentos foi próximo ou superior a $90 \%$ (Figura 4).

Para a massa seca da raiz, verificou-se diferença significativa apenas para a testemunha sem aplicação do herbicida que apresentou valor significativamente maior. Entre os tratamentos não houve diferença significativa. Para a massa seca da parte aérea, observou-se diferença significativa entre os tratamentos, sendo que a utilização de $20 \mathrm{~L} \mathrm{ha}^{-1}$ resultou em menor média, independente do adjuvante utilizado porém semelhante a testemunha com uso do bico hidráulico (Tabela $3)$. 


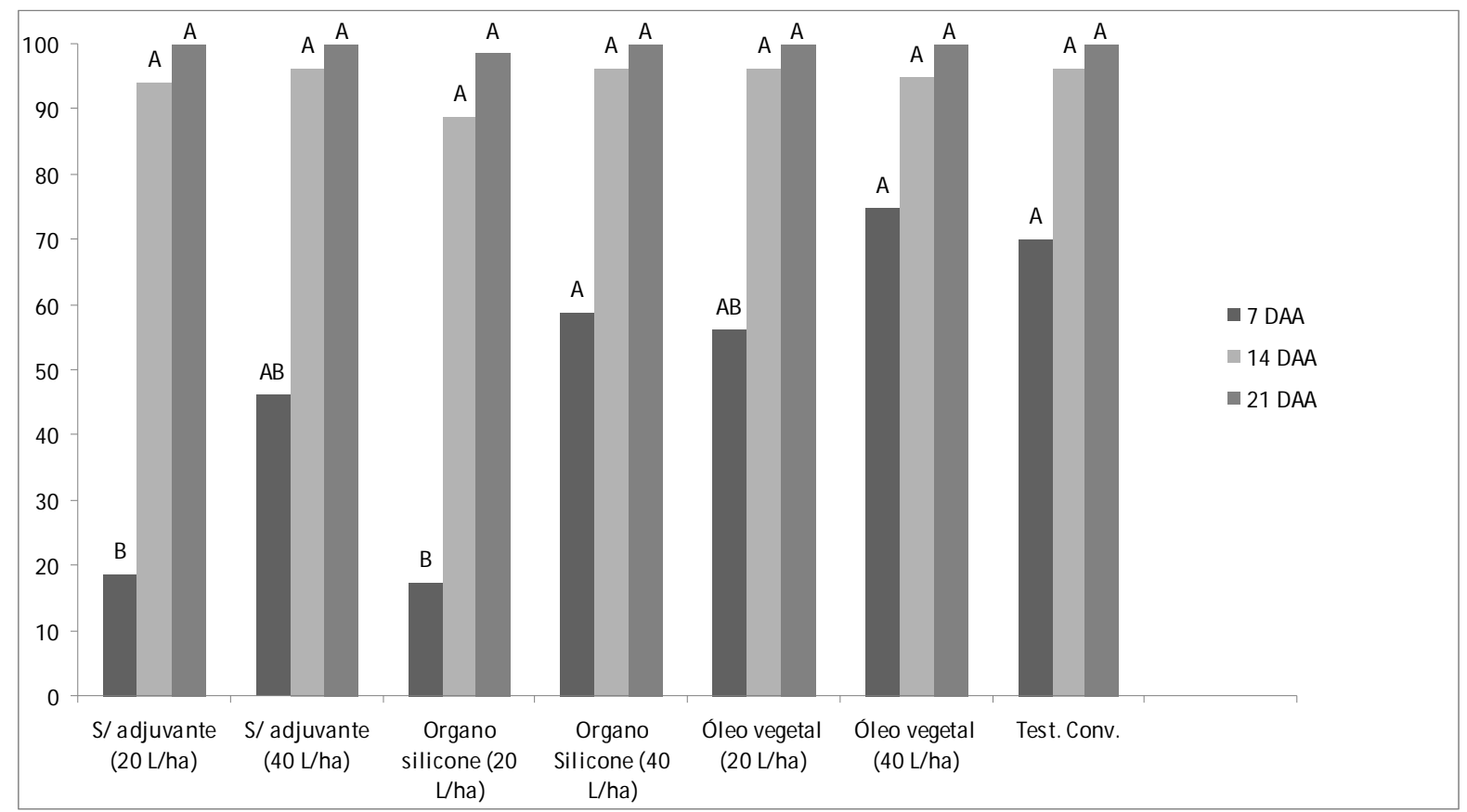

Figura 4. Médias de porcentagem de controle de I. hederifolia aos 7, 14 e 21 DAA. Jaboticabal, 2009.

Tabela 3. Massa seca da parte aérea e raiz de I. hederifolia aos 21 dias após a aplicação.

\begin{tabular}{ccc}
\hline & \multicolumn{2}{c}{ Massa Seca $(\mathrm{g})$} \\
\cline { 2 - 3 } Variável & Parte aérea & Raiz \\
\hline Volume $(\mathrm{V})$ & $0,14 \mathrm{~b}$ & $0,11 \mathrm{a}$ \\
$40 \mathrm{~L}$ & $0,18 \mathrm{a}$ & $0,09 \mathrm{a}$ \\
\hline Adjuvantes (A) & $0,17 \mathrm{a}$ & $0,11 \mathrm{a}$ \\
\hline Ausente & $0,16 \mathrm{a}$ & $0,09 \mathrm{a}$ \\
Org. Silic. & $0,15 \mathrm{a}$ & $0,10 \mathrm{a}$ \\
\hline Óleo vegetal & & \\
\hline Testemunhas (T) & $0,19 \mathrm{~b}$ & $0,08 \mathrm{~b}$ \\
\hline Convencional & $0,72 \mathrm{a}$ & $1,00 \mathrm{a}$ \\
Sem aplicação & $250,53^{* *}$ & $42,86^{* *}$ \\
\hline Test. x Fatores & $257,47^{* *}$ & $61,19 * *$ \\
Entre Test. & $4,82^{*}$ & $0,02^{\mathrm{NS}}$ \\
$\mathrm{F}_{\mathrm{V}}$ & $0,33^{\mathrm{NS}}$ & $0,02^{\mathrm{NS}}$ \\
$\mathrm{F}_{\mathrm{A}}$ & $2,93^{\mathrm{NS}}$ & $0,60^{\mathrm{NS}}$ \\
VxA & 19,71 & 77,6 \\
CV \% & & 0
\end{tabular}

Médias seguidas de mesma letra não diferem entre si, pelo teste Tukey, a $5 \%$ de probabilidade; **: significativo $(\mathrm{P}<0,01)$; *: significativo $(\mathrm{P}<0,05)$; NS: não-significativo; C.V.: coeficiente de variação. 
Assim, não houve diferença de controle da planta daninha com o uso de adjuvantes, e o uso do bico rotativo apresentou a mesma eficiência de controle que o bico hidráulico que foi utilizado como testemunha sobre as plantas de I. hederifolia.

\section{Conclusões}

A adição de adjuvantes à calda de pulverização não promoveu acréscimo no controle de I. hederifolia, assim como não foram constatadas diferenças de controle de $I$. hederifolia entre o bico rotativo Herbi- $4^{\circledR}$ e a ponta hidráulica TT110.01.

O bico rotativo Herbi- $4^{\circledR}$ reduziu consideravelmente o potencial de deriva em relação à ponta hidráulica TT110.01, devido o aumento do diâmetro volumétrico das gotas (DMV), redução da porcentagem de gotas menor que $100 \mu \mathrm{m}$, e maior uniformidade das gotas produzidas, apesar de ter apresentado padrão de distribuição desuniforme.

\section{Referências}

ANTUNIASSI, U. R. Tecnologia de aplicação de defensivos. Revista Plantio Direto, v.15, n. 1, p.17-22, 2006.

BAUER, F. C.; RAETANO, C. G. Distribuição volumétrica de calda produzida pelas pontas de pulverização XR, TP e TJ sob diferentes condições operacionais. Planta Daninha, v.22, n.2, p.275-284, 2004.

CAMARA, F.T. et al. Distribuição volumétrica e espectro de gotas de bicos hidráulicos de jato plano de faixa expandida XR11003. Engenharia Agrícola, v.28, n.4, p.740-749, 2008.

CARBONARI, C.A. et al. Efeito de surfactantes e pontas de pulverização na deposição de calda de pulverização em plantas de grama-seda. Planta Daninha, v.23, n.4, p.725-729, 2005.

CUNHA, J.P.A.R. et al. Avaliação de estratégias para a redução da deriva de agrotóxicos em pulverizações hidráulicas. Planta Daninha, v.21, n.2, p.325-32, 2003.

CUNHA, J.P.A. et al. Espectro de gotas de bicos de pulverização hidráulicos de jato plano e de jato cônico vazio. Pesquisa Agropecuária Brasileira, v.39, n.10, p.977-85, 2004.

CUNHA, J.P.A.R.; ALVES, G.S. Características físico-químicas de soluções aquosas com adjuvantes de uso agrícola. Interciência, v.34, n.9, p.655-659, 2009.

CUNHA, J.P.A.R.; BUENO, M.R.; FERREIRA, M. C. Espectro de gotas de pontas de pulverização com adjuvantes de uso agrícola. Planta Daninha, v.28, n.esp., p.11531158, 2010.

CUNHA, J.P.A.R.; SILVA, R.A.M. Uniformidade de distribuição volumétrica de pontas de pulverização em função da pressão de trabalho e altura da barra. Bioscience Journal, v.26, n.1, p.52-58, 2010.

DI OLIVEIRA, J.R.G. et al. Diferentes diâmetros de gotas e equipamentos para aplicação de inseticida no controle de Pseudoplusia includens. Engenharia Agrícola, v. 30, n. 1, p. 92-99, 2010.

ETHERIDGE, R.E.; WOMAC, A.R.; MUELLER, T.C. Characterization of the spray droplet spectra and patterns of four venturitype drift reduction nozzles. Weed Technology, v.13, n.4, p.765-770, 1999.

FAO. FOOD AGRICULTURE AND ORGANIZATION. Equipo de aplicación de pesticida para uso en agricultura: equipo impulsado mecánicamente. ROMA, 1997. v.2, $150 \mathrm{p}$. 
FLECK, N.G. et al. Controle de papuã (Brachiaria plantaginea) em soja em função da dose e da época de aplicação do herbicida clethodim. Planta Daninha, v. 26, n. 2, p. 375383, 2008.

MATUO, T. Técnicas de aplicação de defensivos agrícolas. Jaboticabal: FUNEP, 1990. 140p.

ORGANIZACIÓN MUNDIAL DE LA SALUD. Material de lucha contra los vetores. Genebra, 1976. 189p.

PERECIN, D. et al. Padrões de distribuição obtidos com bicos TF-VS4, TJ60-11006 e TQ15006 em mesa de prova. Pesquisa Agropecuária Brasileira, v.33, n.2, p.175-82, 1998.

UNE-EN. Maquinaria agrícola y forestal Pulverizadores y distribuidores de fertilizantes líquidos. Protección medioambiental. Parte 2: Pulverizadores hidráulicos de barras para cultivos bajos. 2002. (UNE-EN 12761-2:2002)

VALE, F.X.R. et al. Quant - A software to quantify plant disease severity. In: International Workshop On Plant Disease Epidemiology; The International Society of Plant Pathology, 2001, Ouro Preto, Brazil, Proceedings... vol.8, p. 160.

VIANA, R.G. et al. Distribuição volumétrica e espectro de gotas de pontas de pulverização de baixa deriva. Planta Daninha, v.28, n.2, p.439-446, 2010.

WOMAC, A.R.; MAYNARD, R.A.; KIRK, I.W. Measurement variations in reference sprays for nozzle classification. Trans. ASAE, v.42, n. 2, p.609-616, 1999. 